\title{
Combination of Amlexanox and Forskolin Attenuates Pathological Cardiac Hypertrophy by Subduing Maladaptive Inflammatory Response
}

\author{
Gabriel Adzika ${ }^{1}$, Hongjian Hou ${ }^{1}$, Adebayo Adekunle ${ }^{1}$, Ruqayya Rizvi ${ }^{1}$, Tongtong Ma $^{1}$, \\ Seyram Adzraku², Li Kexue ${ }^{1}$, Qi-Ming Deng ${ }^{3}$, Richard Mprah ${ }^{1}$, Marie Louise Ndzie Noah $^{1}$, \\ Jeremiah Machuki ${ }^{1}$, Stephane Koda ${ }^{1}$, Xianluo $\mathrm{Ma}^{4}$, and Hong Sun ${ }^{1}$ \\ ${ }^{1}$ Xuzhou Medical University \\ ${ }^{2}$ The Affiliated Hospital of Xuzhou Medical University \\ ${ }^{3}$ Shandong University Affiliated Hospital \\ ${ }^{4}$ People's Hospital of Jiawang District
}

July 30, 2020

\begin{abstract}
Background and Purpose: The immune system is implicated in the pathogenesis of pathological cardiac hypertrophy (PCH). However, there is currently no therapeutic intervention to prevent $\mathrm{PCH}$. Here, we aimed at preventing pathological cardiac hypertrophy $(\mathrm{PCH})$ during chronic catecholamine stress via modulating adaptive inflammatory by targeting adenylyl cyclases (ACs) and G protein-coupled receptor kinase 5 (GRK5) in cardiomyocytes and immune cells. Experimental Approach: PCH was induced in mice by chronic isoproterenol injections. In vitro, peritoneal macrophages were challenged with lipopolysaccharide under stress. Further experiments employed the therapeutic interventions Amlexanox and Forskolin to inhibit GRK5 and activate ACs-cAMP, respectively. Cardiac functions were assessed with echocardiography. Inflammatory markers were assessed with ELISA and RT-qPCR (in vivo and in vitro). GRK5 localizations in macrophages were assessed by immunofluorescence, and alterations in protein expression were analyzed with immunoblotting. Histological assessments were done with Masson, H\&E and IHC staining. Key Results: PCH mice had deteriorating cardiac functions and morphological remodeling, accompanied by massive immune cell infiltrations. Similarities were observed proinflammatory markers upregulation, as were IL-10 found downregulated both in vivo and in vitro. However, the combination of Amlexanox and Forskolin modulated adaptive inflammatory responses and also maintained proper cardiac morphology and function. The single therapies of neither Amlexanox nor Forskolin were able to attain the aforementioned with much efficacy as their combination therapy. Conclusion: The combination therapy of ALX and FSK has the therapeutic potential of preventing the occurrence of pathological cardiac hypertrophy during CCS by modulating adaptive inflammatory responses while maintaining normal cardiac function.
\end{abstract}

\section{Combination of Amlexanox and Forskolin Attenuates Pathological Cardiac Hypertrophy by Subduing Maladaptive Inflammatory Response}

Running title: Pathological Cardiac Hypertrophy therapeutic intervention

Gabriel Komla Adzika ${ }^{1}$, Hongjian Hou ${ }^{1}$, Adebayo Oluwafemi Adekunle ${ }^{1}$, Ruqayya Rizvi ${ }^{2}$, Tongtong $\mathrm{Ma}^{1}$, Seyram Yao Adzraku ${ }^{3}$, Li Kexue ${ }^{1}$, Qi-Ming Deng ${ }^{4}$, Richard Mprah ${ }^{1}$, Marie Louise Ndzie Noah ${ }^{1}$, Jeremiah Ong'achwa Machuki ${ }^{1}$, Stephane Koda ${ }^{5}$, Xianluo Ma ${ }^{6}$, Hong Sun ${ }^{1,2}$

\section{Affiliations}


1. Department of Physiology, School of Basic Medical Sciences, Xuzhou Medical University, Xuzhou,221004, Jiangsu, China.

2. Xuzhou Medical University, Xuzhou, 221004, Jiangsu, China.

3. Department of Hematology, Key Laboratory of Bone Marrow Stem Cell, The Affiliated Hospital of Xuzhou Medical University, Xuzhou 221002, China

4. The Key Laboratory of Cardiovascular Remodeling and Function Research, Chinese Ministry of Education, Chinese National Health Commission and Chinese Academy of Medical Sciences, The State and Shandong Province Joint Key Laboratory of Translational Cardiovascular Medicine, Department of Cardiology, Qilu Hospital of Shandong University

5. Department of Pathogenic Biology and Immunology, Laboratory of Infection and Immunity, Xuzhou Medical University, People's Republic of China

6. People's Hospital of Jiawang District, Internal Medicine - Cardiovascular Department, Xuzhou, Jiangsu, China.

Address correspondence to:

Prof. Hong Sun (MD, Ph.D.). Department of Physiology, Xuzhou Medical University, 209 Tongshan Road,Xuzhou,221004, Jiangsu, China. Phone: +86-516-83262618, Fax: +86-516-83262858. Email: sunh@xzhmu.edu.cn.

\section{Abstract}

Background and Purpose: The immune system is implicated in the pathogenesis of pathological cardiac hypertrophy $(\mathrm{PCH})$. However, there is currently no therapeutic intervention to prevent PCH. Here, we aimed at preventing pathological cardiac hypertrophy $(\mathrm{PCH})$ during chronic catecholamine stress via modulating adaptive inflammatory by targeting adenylyl cyclases (ACs) and G protein-coupled receptor kinase 5 (GRK5) in cardiomyocytes and immune cells.

Experimental Approach: PCH was induced in mice by chronic isoproterenol injections. In vitro, peritoneal macrophages were challenged with lipopolysaccharide under stress. Further experiments employed the therapeutic interventions Amlexanox and Forskolin to inhibit GRK5 and activate ACs-cAMP, respectively. Cardiac functions were assessed with echocardiography. Inflammatory markers were assessed with ELISA and RT-qPCR (in vivo and in vitro). GRK5 localizations in macrophages were assessed by immunofluorescence, and alterations in protein expression were analyzed with immunoblotting. Histological assessments were done with Masson, H\&E and IHC staining.

Key Results: PCH mice had deteriorating cardiac functions and morphological remodeling, accompanied by massive immune cell infiltrations. Similarities were observed proinflammatory markers upregulation, as were IL-10 found downregulated both in vivo and in vitro. However, the combination of Amlexanox and Forskolin modulated adaptive inflammatory responses and also maintained proper cardiac morphology and function. The single therapies of neither Amlexanox nor Forskolin were able to attain the aforementioned with much efficacy as their combination therapy.

Conclusion: The combination therapy of ALX and FSK has the therapeutic potential of preventing the occurrence of pathological cardiac hypertrophy during CCS by modulating adaptive inflammatory responses while maintaining normal cardiac function.

Keywords: Chronic Catecholamine Stress, Inflammation, Pathological Cardiac Hypertrophy, Amlexanox, Forskolin

\section{Bullet point summary}

What is already known

- Amlexanox attenuates cardiomyocyte hypertrophy and regulates anti-inflammatory responses

- Forskolin induces ACs-cAMP-dependent immunoregulation besides facilitating cardiac contractions 
What this study adds

- Amlexanox or Forskolin single therapies do not exhibit much potency in adaptive immunoregulation, preservation of cardiac function

- The synergy of Amlexanox and Forskolin keeps the heart healthy and functional during CCS

Clinical significance

Amlexanox and Forskolin combination therapy may be a potential intervention for $\mathrm{PCH}$ prevention

\section{Introduction}

The elevated level of circulating catecholamines during chronic stress is a hallmark for the initiation and progression of adverse cardiac remodeling (Paur H et al., 2012; Adzika GK et al., 2019). Pathological cardiac hypertrophy $(\mathrm{PCH})$ is the irreversible resultant cardiomyopathy if there are no timely preventive measures to subdue the excessively firing of neurohormonal stimuli during chronic stress. Left ventricular (LV) diastolic dysfunction is a clinical characteristic of patients having $\mathrm{PCH}$. This heart malfunction is a result of the abnormal increase in heart size with excessively thickened ventricular walls which, are stiffer due to the massive deposition of collagen. The typical myocardium architecture becomes distorted. The LV becomes dilated, and the heart loses its ability to rapidly replenish blood for the next ejection (Xie M et al., 2013; Hartupee J et al., 2016). The heart harbors resident macrophages which crosstalk between cardiomyocytes and other cellular factions such as fibroblasts to ensure myocardial homeostasis. However, this crosstalk implicates the immune system in playing a pivotal role in the pathological remodeling of the heart (Hartupee J et al., 2016; Kong P et al., 2014). Cardiac resident macrophages are stimulated by cardiac danger-associated molecular patterns (cDAMPs), cardiac troponin, and myosin that being released by necrotic cardiomyocytes. These resident macrophages elicit proinflammatory responses in an attempt to curb further myocyte necrosis (Epelman S et al., 2014; Heidt T et al., 2014). This immune response is aggravated under chronic catecholamine stress (CCS) conditions and a biased hyperactive inflammatory result instead. The secretion of Interleukin (IL) 1 $\beta$, IL-2, IL-6, tumor necrosis factor-alpha (TNF $\alpha$ ), and interferon-gamma $(\mathrm{IFN}-\gamma)$ are excessively while IL-10 secretion becomes downregulated. The aforementioned exacerbates the myocyte necrosis, prolongs myofibroblast activation and increases interstitial fibrosis markedly (Kong P et al., 2014; Hulsmans M et al., 2016). Interestingly, the innate immune response elicited when challenged by cardiac troponin and myosin during CCS is very similar with lipopolysaccharides (LPS)-challenged macrophages under the same stress conditions (Laukova $\mathrm{M}$ et al., 2018).

$\beta_{1}$-adrenergic receptor $\left(\beta_{1} \mathrm{AR}\right)$ and $\beta_{2}$-adrenergic receptor $\left(\beta_{2} \mathrm{AR}\right)$ expressed in the heart mediate the neuroendocrine stimulates the regulate cardiac function. $\beta_{1} \mathrm{AR}$ modulates chronotropic and inotropic via the typical stimulatory $\mathrm{G}$ protein $\left(\mathrm{G}_{\alpha \varsigma}\right)$ - adenylyl cyclases (ACs) - cyclic adenosine monophosphate (cAMP) pathway. The pleiotropic nature of $\beta_{2} A R$ enables it to traffick stimuli via $G_{\alpha \varsigma}$ or $G_{\alpha \iota}$ in cardiomyocytes, and immune cells. Also, a mounting of evidence indicates that $\beta_{2} \mathrm{AR}$ mediates maladaptive stimuli signaling during cardiovascular diseases (CVDs) (Paur H et al., 2012; Adzika GK et al., 2019; Laukova M et al., 2018). These suggests exploring the post- $\beta_{2} \mathrm{AR}$ proteins and kinases may have therapeutic potentials of attenuating the occurrence of PCH. Furthermore, a negative correlation exists between ACs and GRKs in healthy and failing cardiac and immune systems (Adzika GK et al., 2019). Therefore, this study focuses on ACs and G protein-coupled receptor kinases (GRKs) to exploit their possible therapeutic potentials.

ACs in generally synthesize cAMP. AC isoform AC7 is expressed explicitly in immune cells. It induces antiinflammatory response via increase cAMP synthesis. ${ }^{2}$ While expression of the isoforms AC5 and AC6 are cardiac specific (Li Y et al.,2012). cAMP is essential for maintaining homeostasis in the immune system and proper cardiac function of the heart. Besides the activation of protein kinase A (PKA) in cardiomyocytes and immune cells, cAMP adaptively modulates the activities nuclear factor of activated T-cells (NFATs) and NF-kB (Kipanyula MJ et al, 2013; Pereira L et al., 2015; Murphy JG et al., 2019; Gerlo S et al., 2011). However, during CCS, the ACs are uncoupled from $\mathrm{G}_{\alpha \varsigma}$, terminating the synthesis of cAMP and abolishing its modulation of proper cardiac and immune functions. 
GRKs modulates the expression of $\beta_{2}$ AR. GRK2 isoform mainly desensitizes and internalizes $\beta_{2}$ AR during catecholamine stress. Conversely, GRK5 can translocate from the plasma membrane into the nucleus. As such, its upregulation facilitates its entry into the nucleus to maladaptively phosphorylate transcription factors (TFs) that elicits adverse immune responses and pathological cardiac remodeling (Fig. 1) (Patial S et al., 2011; Gravning J et al., 2013; Hullmann JE et al., 2014). GRK5 blockade impedes the non-canonically activation of immune and cardiac transcription factors, NFATs, myocyte enhancer factor 2(MEF2), GATA4, Csx/Nkx2-5, and NF-kB. Thus, GRK5 inhibition attenuates cardiac hypertrophy and, to some extent, maladaptive immune response during stress (Hullmann JE et al., 2014; Quan MY et al., 2019).

Nonetheless, there is no evidence that neither cardiac function was improved nor attenuation of hyperactive immune responses when GRK5 was knockout or inhibited with amlexanox (ALX) in an attempt to prevent $\mathrm{PCH}$ during CCS. Also, besides the known roles AC5, AC6 and AC7, there have been no attempts to directly stimulate their activities by using Forskolin (FSK) to adaptively modulate the immune response and cardiac function to prevent $\mathrm{PCH}$.

Herein, we show that the combination therapy of ALX and FSK can maintain proper cardiac function while sustaining immune homeostasis. Our data from preliminary in vitro experiments revealed that the combination treatment of peritoneal macrophages $\left(\mathrm{PM}_{\mathrm{F}}\right)$ with ALX and FSK after being challenged with Isoproterenol (ISO) and LPS, attenuated induction hyperactive proinflammatory responses. However, neither single treatments with ALX nor FSK were able to attain this as much. These were translated in vivo to curb inflammatory responses from exacerbating cardiomyocyte necrosis and adversely remodeling the heart into PCH during CCS.

\section{Materials and Methods}

\section{Experimental animals and models}

Male FVB wild-type (WT) mice aged between 8-12 weeks were randomized, grouped, and used for both in vitro and in vivo experiments in this study. $\mathrm{PCH}$ in vivo model was made by subcutaneous injection of Isoproterenol (160504; Sigma) 0.5mg/100g/day for 40 days. An equivalent volume of the placebo (5\% $\mathrm{v} / \mathrm{v}$ dimethyl sulfoxide (DMSO)) was injected subcutaneously to the vehicle (Vhl) group. Their littermates without any handling were included in the study and designated as the control (Ctrl) group.

$\mathrm{PCH}$ preventive models were made by intraperitoneal injection of a GRK5 inhibitor drug, Amlexanox (ab142825; Abcam) 2.5mg/100g/day or, and ACs direct activator Forskolin (1099; Tocris Bioscience, UK) $0.5 \mathrm{mg} / 100 \mathrm{~g} /$ day, simultaneously with ISO $0.5 \mathrm{mg} / 100 \mathrm{~g} /$ day from day 1 to day 40 (Supplementary Fig. S1).

\section{Echocardiography}

Mice were mildly sedated with $0.5 \%$ isoflurane and fixed with tape and echo gel on a warm ECG platform. Global cardiac function was assessed in M-mode with a Vevo 2100 Ultrasound system (VisualSonics, Toronto, Canada). At end-systole and end-diastole, the interventricular septal wall and posterior wall thicknesses, as well as left ventricle internal diameters, were evaluated from the left parasternal longitudinal axis view. Ejection fraction (EF) and fractional shorten (FS) were calculated as described (Jia D et al., 2019).

\section{Immune cells isolation and culture}

$\mathrm{PM}_{\mathrm{F}}$ were isolated from male FVB WT mice as previously described (Ray A et al., 2010), but with some modifications. A euthanized mouse fully submerged in $75 \%$ ethanol was fixed on its back, and the inner skin lining exposed with scissors and forceps. $8-10 \mathrm{ml}$ of $37^{\circ} \mathrm{C}$ PBS containing $3 \%$ fetal bovine serum (FBS) was carefully injected into the peritoneal cavity, and the peritoneum was massaged for 5 min. The cell suspension was then collected from the peritoneum, centrifuged at $1500 \mathrm{rpm}$ for $10 \mathrm{~min}$. The pellet of cells was resuspended in DMEM high glucose (Gbico; Thermo Fisher Scientific) containing 10\% FBS and cultured for four days. After trypsinization, cells were washed twice with PBS and incubated for 30 min with the following monoclonal antibodies: APC anti-mouse F4/80 (123116; BioLegend), FITC antimouse/human CD11b (101206; BioLegend) for macrophages identification and characterization using flow 
cytometry (LSRFortessa; BD Biosciences).

For the ALX treatment group, cultured $\mathrm{PM}_{\mathrm{F}}$ were pre-treated for an hour with $35 \mu \mathrm{M} / \mathrm{ml}$ of ALX and challenged with $10 \mu \mathrm{M} / \mathrm{ml}$ of ISO and, or $1 \mu \mathrm{g} / \mathrm{ml}$ LPS. Next, treatments with $35 \mu \mathrm{M} / \mathrm{ml}$ of ALX and, or $10 \mu \mathrm{M} / \mathrm{ml} \mathrm{FSK}$ followed. The treated $\mathrm{PM}_{\mathrm{F}}$ were incubated at $37^{\circ} \mathrm{C}$ and $5 \% \mathrm{CO}_{2}$ for $24 \mathrm{~h}$. The supernatants from the culture were collected and stored in $-80^{\circ} \mathrm{C}$ for cytokine and cAMP assays.

\section{Inflammatory cytokines and cAMP assay}

Plasma in vivo models and cell culture media supernatants from treated macrophages were used to assess the concentrations of inflammatory cytokines (IL-1 $\beta$, IL-6, TNF $\alpha$, IL-10) and cAMP by Enzyme-linked immunosorbent assay (ELISA). IL-1 $\beta$ (ab197742; Abcam), IL-6 (ab222503; Abcam), TNF $\alpha$ (ab208348; Abcam), IL-10 (ab255729; Abcam) and cAMP (JL13362; Jianglai Bio, China) ELISA kits were used as per the manufacturer's instructions.

\section{RNA extraction and real-time qPCR}

Total RNA was extracted from ventricular apical myocardium using TRIzol (15596026; Life technologies). cDNA was synthesized from 1ug of RNA using a Reverse Transcription Reagent kit according to the manufacturer's instruction (FSQ107; Toyobo). The obtained cDNAs were amplified by semiquantitative RT-PCR using SYBR Green Master Mix (Q111-02; Vazyme). The primer sequences used are listed here ((Supplementary Table S1). The relative gene expressions were normalized to the of GAPDH and comparisons were done with the ddCt method as described (Gold JI et al., 2013).

\section{Immunofluorescence staining}

Cultured and pre-treated $\mathrm{PM}_{\mathrm{F}}$ were fixed and permeabilized with pre-chilled methanol-acetone of ratio 1:1. Non-specific antibody binds were blocked with $1 \%$ BSA in PBS for $1 \mathrm{~h} . \mathrm{PM}_{\mathrm{F}}$ were then incubated with GRK5 primary antibody (ab64943; Abcam) at $4^{\circ} \mathrm{C}$ overnight, washed with PBS, and probed with R-PE-conjugated secondary antibody (SA00008-2; Proteintech) at room temperature for $1 \mathrm{~h}$. Next, cells were washed with PBS, conditioned with $0.5 \%$ BSA in Hanks' balanced salt solution (HBSS), and the cytoplasmic membranes were stained with cholera toxin B (CTxB) (C34775, ThermoFisher Scientific) for 30 min at $4^{\circ} \mathrm{C}$. DAPI nuclei stainings were done and followed by imagings. ImageJ was used to measure cytoplasmic and nuclei regions of interest. The measured values were corrected for background signal, and nucleic and cytoplasmic GRK5 expression ratios were calculated.

\section{Cardiomyocytes hypertrophy, interstitial fibrosis and immune cells infiltration}

Whole hearts and ventricles were harvested, rinsed of blood with pre-chilled PBS and were fixed in $3 \%$ formalin. The heart specimens were then embedded in paraffin and sliced into $4 \mu \mathrm{m}$ thick sections. Haematoxylin and eosin (H\&E), Masson's trichrome, and CD68 immunohistochemical (IHC) stainings were performed on tissue sections. The diameter of cardiomyocytes from the H\&E stained sections was measured to determine the increase in myocyte size. The trichrome stained sections were used to evaluate the percentage of collagen deposit by dividing the collagen area with the total myocardial area and multiple by 100 , as described earlier (Jia D et al., 2019).

CD68 IHC staining was done following the general protocol with a few optimizations. In brief, after deparaffination, heat-induced antigen retrieval was done using citrate butter. Non-specific antibody binds were prevented by flooding the slides with $\mathrm{H}_{2} \mathrm{O}_{2}$ for $10 \mathrm{~min}$ and followed by $3 \%$ BSA for $30 \mathrm{~min}$. The tissue sections were then incubated overtime at $4^{\circ} \mathrm{C}$ with CD68 primary antibody (ab955, Abcam) diluted in $1 \%$ BSA. The tissues were incubated with biotinylated goat anti-rabbit IgG and Streptavidin peroxidase for 25 min each, followed by DAB staining and hematoxylin counterstaining. Imaging of tissue slides was done at x400 magnification and analyzed with ImageJ (1.52a version; National Institute of Health, USA) was utilized in these analyses.

\section{Western Blot}


The apex of the ventricular myocardia were homogenized in a lysis buffer containing phosphatase and proteinase cocktail inhibitor, in the ratio 100:1:1 respectively. Lysates of normalized concentrations and treated with reducing agents were denatured at $100^{\circ} \mathrm{C}$ for $10 \mathrm{~min}$ and separated on SDS-PAGE gel. The protein bands were transferred at 100V for $1 \mathrm{~h}$ onto polyvinylidene fluoride (PVDF) membranes and blocked with $1 \%$ bovine serum albumin in Tris-buffered saline and Tween 20. The primary antibodies employed in this study are as follows: $\beta_{1}$ AR (ab3442; Abcam), $\beta_{2}$ AR (ab182136; Abcam), MEF2 (ab64644; Abcam), GRK5 (ab64943; Abcam) GATA4 (ab84593; Abcam), NFAT (ab25916; Abcam), AC5 (PAC-501AP; FabGennix), AC6 (PAC-601AP; FabGennix), AC7 (PAC-701AP; FabGennix), ANP (sc-515701; Santa Cruz Biotechnology), BNP (sc-271185; Santa Cruz Biotechnology), GRK2 (sc-13143; Santa Cruz Biotechnology), $p$ ERK1/2 (4370; Cell Signaling Technology), ERK1/2 (9102S; Cell Signaling Technology), $p$ NF- $\varkappa$ B (3033T; Cell Signaling Technology), NF- $x$ B (8242T; Cell Signaling Technology) and GAPDH (10494-1-AP; Proteintech). Blots imaging was done using an enhanced chemiluminescence (Tanon, Shanghai, China). Protein bands were quantified and normalized with their respective GAPDH expressions.

\section{Statistical analysis}

The comparisons made involving two groups were made using the Student's t-test. ANOVA was used to comparing data of multiple groups, and post hoc analysis was done using Tukey's multiple comparisons test. Kaplan-Meier estimator was used to estimate survival distributions. P values scoring less than 0.05 were deemed significant. GraphPad Prism (Prism Version 8.0.2; Graph Pad Prism Software Inc., San Diego, USA) was used for all the statistical analyses.

\section{Results}

\section{Cardiac and Immune Functional Proteins are altered during CCS}

The results obtained from mRNAs and protein levels demonstrates alteration in the expressions of $\beta$ ARs, ACs, GRK2 and GRK5 in the myocardium during CCS compared with under normal physiological conditions (Supplementary Fig. S2a and S2b). Specifically, $\beta$ ARs, AC5, and AC7 were found depleting while AC6, GRK2 and GRK5 were upregulated in the PCH mice hearts.

Overexpression of GRK5 in the hearts of PCH mice must have facilitated its translocation and localization to the nucleus of cardiomyocytes to mediate the exacerbation of $\mathrm{PCH}$ as evident by both atrial natriuretic peptide (ANP) and brain natriuretic peptide (BNP) upregulations (Supplementary Fig. S2a and S2b) (Hullmann JE et al., 2014).

cAMP concentrations assessed by ELISA to ascertain the impact of AC5, AC6 and AC7 alterations showed no significant differences between Ctrl and Vhl mice. However, cAMP concentration in PCH mice was significantly decreased compared with both Vhl and Ctrl mice (Supplementary Fig. S2c).

Also, extracellular signal-regulated kinase (ERK1/2) and cardiac hypertrophy and inflammatory TFs; GATA4, nuclear factor of activated T cells (NFAT), myocyte enhancer factor-2 (MEF2) and nuclear factor kappa-light-chain-enhancer of activated $\mathrm{B}$ cells $(\mathrm{NF}-x \mathrm{~B})$ were all comparably fairly expressed in the hearts of Ctrl and Vhl mice but overexpressed in PCH mice hearts (Supplementary Fig. S3).

\section{Necrotic cardiomyocytes and LPS elicits similar immunogenic response during CCS}

The immune system is implicated in the exacerbation of cardiac hypertrophy due to its hyperactive inflammation response to the cDAMPs, cardiac myosin and troponin released by necrotic cardiomyocytes (Kong P et al., 2014; Hulsmans M et al., 2016). ${ }^{5,8}$ Herein, we provide evidence that the inflammatory responses elicited by cardiomyocyte necrosis during CCS are very similar to the immune response invoked by LPS under the same stress conditions. Profiling of alterations in the inflammatory markers IL-1 $\beta$, IL-6, IL-10, $\mathrm{TNF} \alpha, \mathrm{IFN} \gamma$, and $\mathrm{NF}-\varkappa \mathrm{B}$ was done using RNAs isolated directly from the apical myocardium for RT-qPCR analysis. Results from the analysis showed the proinflammatory cytokines IL- $1 \beta$, IL-6, TNF $\alpha$, IFN $\gamma$, and $\mathrm{NF}-\varkappa \mathrm{B}$ the prototypic inflammatory pathway were significantly overexpressed in the PCH mice in comparison to Ctrl and Vhl mice. Meanwhile, PCH mice had their anti-inflammatory cytokine, IL-10, significantly 
downregulated on comparing its expression with Ctrl and Vhl mice (Fig. 2a). Sera from these mice groups were assessed for the same inflammatory cytokines with ELISA, and the results obtained showed similarities in expression trends of both the pro and anti-inflammatory cytokines on comparing the mRNAs expressions with sera cytokine concentrations (Fig. 2a and 2b).

To compare the immunogenic response between necrotic cardiomyocyte and LPS during CCS, $\mathrm{PM}_{\mathrm{F}}$ were challenged with ISO and LPS for $24 \mathrm{~h}$ and obtained supernatants were analyzed with ELISA. Intriguing, the resulted revealed under CCS, LPS invokes a hyperactive inflammatory response like what was observed in $\mathrm{PCH}$ mice that had marked myocyte necrosis (Fig. 2b). The hyperactive immune response elicited by LPS under CCS was observed elsewhere as well (Laukova M et al., 2018).

\section{Combination of ALX and FSK minimized LPS-induced inflammatory response during CCS with much efficacy}

The inhibitory effect of ALX on GRK5, which participates in maladaptive activities in cardiac and immune cells, was used to treatment $\mathrm{PM}_{\mathrm{F}}$ challenged with LPS under CCS condition to attenuate the invocation of a hyperactive immune response. FSK was also utilized to directly stimulate ACs to synthesize cAMP, which has a positive effect on chronotropic and inotropic in cardiomyocytes and an anti-inflammatory effect in immune cells to modulate $\mathrm{PM}_{\mathrm{F}}$ response during CCS. Also, the stressed $\mathrm{PM}_{\mathrm{F}}$ challenged with LPS were also treated with the combination of ALX and FSK.

Results from ELISA analysis of the supernatants from all groups are demonstrated in (Fig. 3). Although all the therapeutic invention groups (ALX, FSK, and ALX with FSK) decreased the extent of immune hyperactivation of $\mathrm{PM}_{\mathrm{F}}$ elicited by LPS during CCS, the single treatment of ALX had the least efficacy compared with FSK single therapy and their combine therapy. More than the single treatment with FSK after the $\mathrm{PM}_{\mathrm{F}}$ had been challenged with LPS under stress, the combination treatment with ALX and FSK was effective in minimizing IL-1 $\beta$, IL-6, and TNF $\alpha$ (Fig. 3a-3c) secretions while keeping IL-10 upregulated (Fig. 3d).

\section{Combination of ALX and FSK inhibited GRK5 expression and its nuclear activities in $\mathrm{PM}_{\mathrm{F}}$}

Nuclear translocation of immune cells GRK5 in $\mathrm{PM}_{\mathrm{F}}$ was investigated with immunofluorescence (IF). After treatment of $\mathrm{PM}_{\mathrm{F}}$-LPS-ISO with ALX, FSK, and their combination overtime, IF staining for GRK5 (R-PE dye), nuclei (DAPI), and cytoplasmic membrane (CTxB - Alexa Fluor $\mathrm{R} 488$ Dye) was done. Imaging showed that without any therapeutic interventions, GRK5 was upregulated in PM $_{\mathrm{F}}$-LPS-ISO. ALX single therapy inhibited GRK5 expression significantly and blocked its nuclear translocation activities. Meanwhile, the FSK single treatment had an insignificant effect on GRK5 expression and its nuclear translocation activities. As such, the FSK and ALX combination did just as excellent as the ALX single therapy (Fig. 4).

\section{Combination of ALX and FSK preserved cardiac function during CCS}

Cardiac function assessed at the end of animal models using echocardiogram demonstrated cardiac function deteriorated during CCS. Compared with the PCH mice and ALX single therapy, FSK single therapy improved cardiac function to some extent, but it was accompanied by arrhythmias. However, the combination of ALX and FSK preserved cardiac function most effectively compared to the single therapy groups (Fig. 5a-5c, Supplementary Fig. S4 and Table S2). There was no statistical significance upon comparing cardiac functions of mice from the PCH-preventive combine therapy group with Ctrl and Vhl mice. The Vhl mice group is representing the Ctrl mice group as well in results illustrations due to similarity in their data.

A further critical assessment of molecular alterations in the apical myocardium across all groups demonstrated that overall, the combination of ALX and FSK preserved cardiac function during CCS by normalizing expressions of the proteins and TFs that play critical roles in cardiac function and immune response modulation.

Among the therapeutic groups, $\beta_{1}$ AR depletion was minimized by the combined therapy, while $\beta_{2}$ AR expression was somewhat maintained across all the groups. Compared to the Vhl and PCH mice, the expression of 
AC5 and AC7 were mostly normalized by the combined therapy, although FSK single treatment did better against ALX single treatment. AC6 was still observed increasing its expression in stress intensity-dependent manner across groups. Even though ALX alone inhibited GRK5 activities, it had no inhibitory effect on GRK2; neither did only FSK. FSK single treatment also minimized the upregulation of GRK5 but not as effective as ALX single treatment and their combined treatment. The combination therapy again was the best in regulating ANP and BNP expressions during CCS (Fig. 6a). The normalization of ACs by the combination therapy was translated into normalized cAMP concentrations as well (Fig. 6b).

Intriguingly, despite the inhibition GRK5 by the ALX single treatment, ERK1/2 was maladaptively engaged, and the TFs; GATA4, NFAT, MEF2, and NF- $x$ B were upregulated just like in PCH mice. FSK single therapy did better at modulating the overexpression of these cardiac and inflammatory TFs, but the ALX and FSK combination therapy did best (Fig.7)

\section{Combination of ALX and FSK attenuated CCS-induced PCH}

Morphometric assessment of whole hearts showed that PCH mice had an increase in their heart size as by their heart weight (HW) and body weight (BW) ratio. Comparing the therapeutic groups with the Vhl and PCH groups, ALX alone and its combination with FSK did better at keeping the HW/BW ratio healthy than FSK single therapy (Fig. 8a and Supplementary Table S2). Detailed morphological analysis of longitudinal and transverse tissue sectionings using H\&E and trichrome staining revealed hearts PCH mice had dilated left and right heart chambers, marked collagen deposits, and abnormal hypertrophied cardiomyocytes (Fig. 8a8c). Again, although ALX effectively attenuated cardiomyocyte hypertrophy, it failed to prevent interstitial collagen deposits. Also, FSK alone was not very efficient in attenuating neither myocyte hypertrophy, nor collagen deposit as its combination with ALX did (Fig. 8d and 8e).

\section{Combination of ALX and FSK minimized immune cells infiltration into myocardium and sys-} temic inflammatory response

Ascertaining the extent of mononuclear immune cells infiltrating the myocardium in response to cardiomyocyte necrosis was done CD68 IHC staining. As expected, PCH mice an enormous infiltration immune cell. Comparatively, the FSK single therapy decreased the infiltration of immune cells than the ALX single therapy did. Nonetheless, their combination was effective in minimizing macrophages and other mononuclear cell infiltration into the myocardium (Fig. 9a).

Inflammatory cytokines expression evaluated with ELISA illustrated that among the PCH-preventive therapeutic groups, the FSK and ALX combination therapy had the most efficacy in maintaining a close homeostatic gap between proinflammatory and anti-inflammatory responses during CCS. The secretions of IL-1 $\beta$, IL-6, and TNF $\alpha$ by macrophages in response to necrotic cardiomyocytes were moderate in the mice treated with the combination therapy (Fig. 9b-9d), while IL-10 was upregulated in these mice (Fig. 9e). Treatment with only FSK did better than the treatment with only ALX in the attempt to prevent a hyperactive immune response.

\section{Discussion}

In this study, we demonstrated that, although $\beta_{2} \mathrm{AR}$ is crucial for mediating stimuli in cardiac and immune cells for their proper functioning during CCS (Kim MH et al., 2014), its effector $\mathrm{G}_{\alpha \varsigma}-\mathrm{ACs}$ and receptor kinase - GRKs expressions are altered under extreme stress. AC5 and AC6 expressed in cardiomyocytes exhibited a negative correlation under stress. While AC5 was being depleted in a stress intensity-dependent manner, AC6 was being upregulated in the same manner. This also goes to prove that AC6 mainly handles stress besides calcium channel modulation (Wu YS et al., 2017; Tang T et al., 2008). Like AC5, the immune cells-specific isoform AC7 was also downregulated in $\mathrm{PCH}$ mice. The decrease in the expression of AC5 and AC7 in PCH mice decrease their cAMP concentration level. This implied a decrease in cardiac function and the abolishment of cAMP-dependent anti-inflammatory effects on immune cells (Paur H et al., 2012; Raker VK et al., 2016). The downregulation of cAMP also terminated its adaptive regulation of the TFs; NFAT, MEF2, and NF- $x$ B in both immune cells and cardiomyocytes (Kipanyula MJ et al, 2013; Pereira L et al., 
2015; Murphy JG et al., 2019; Gerlo S et al., 2011; Raker VK et al., 2016).

GRKs are observed to be fairly expressed in Ctrl and Vhl mice but, they are overexpressed in PCH mice to phosphorylate, desensitize and down-regulate hypersensitize $\beta$ ARs. GRK2 typically phosphorylated $\beta$ ARs to desensitize the receptor (Premont RT et al., 2007). Meanwhile, mounting evidence has shown that upregulated GRK5 facilitates a non-canonical GPCR-independent stimulus signaling that progresses adverse cardiac hypertrophy. As previously reported, besides GRK5 upregulation, we also found the overexpression of ANP, BNP and ERK1/2, and TFs: GATA4, NFAT, MEF2, and NF- $x$ B in the PCH mice. The ability of GRK5 to translocate from the cytosol into the nuclei enables it to directly induce nuclear activities by phosphorylating the inflammatory and myocyte TFs (Hullmann JE et al., 2014; Martini JS et al., 2008; Islam KN et al., 2013; Sorriento D et al., 2018). Therefore, GRK5 induces both excessive myocyte hypertrophy and necrosis, and proinflammatory response.

To set the basis for our latter translational in vivo models, we first investigated and compared the immune responses elicited by DAMPs from necrotic cardiomyocytes and LPS, both under chronic stress. The excessive cardiomyocytes necrosis occurring in the hearts of $\mathrm{PCH}$ mice induced increased secretions of proinflammatory cytokines, IL-1 $\beta$, IL-6, TNF $\alpha$, and IFN $\gamma$, while the anti-inflammatory cytokine IL-10 was dampened (Fig. 2a). Although we are not the first to demonstrate the hyperactive inflammatory responses of immune cells to either cDAMP or LPS during CCS (Laukova M et al., 2018; Zimmer A et al., 2019), we are to first to profile and compare the two to show the similarity in their proinflammatory and anti-inflammatory cytokine expressions (Fig. 2b). It could be argued that LPS still induces a proinflammatory response from the macrophages in the absence of CCS. Nonetheless, our obtained data, as well as other researchers, have suggested that the proinflammatory responses induced by LPS are heightened during stress than at the physiological state (Laukova M et al., 2018; Liu YZ et al., 2017; Maydych, V, 2019).

Interestingly, hyperactivation and modulation of the inflammatory response by necrotic cardiomyocytes and LPS are mediated by GRK5 (Patial S et al., 2011; Packiriswamy N et al., 2015). This implied inhibiting GRK may exert an anti-inflammatory effect. As such, we hypothesized that inhibiting GRK5 in $\mathrm{PM}_{\mathrm{F}}$ while directly stimulating ACs to synthesis cAMP to adaptively regulate inflammatory TFs may attenuate the hyperactive response of $\mathrm{PM}_{\mathrm{F}}$ to LPS during chronic stress.

Herein, we explored the inhibitory effects of Amlexanox on GRK5. Also, we utilized Forskolin to stimulate AC-cAMP synthesis, independent of $\beta$ ARs-Gas directly. Both were done in attempts to halt the maladaptive inflammatory response of $\mathrm{PM}_{\mathrm{F}}$ to LPS during CCS. The data obtained from this in vitro experiment supported our earlier hypothesis, along with some unexpected outcomes. ALX single treatment of the LPSchallenged stressed $\mathrm{PM}_{\mathrm{F}}$ was unable to effectively attenuated its hyper proinflammatory response. However, the dosage used in the study was within the range that had been reported earlier to halt inflammatory response. ${ }^{18}$ Comparatively, FSK single treatment performed better than the ALX. Meanwhile, treatment of the LPS-challenged stressed $\mathrm{PM}_{\mathrm{F}}$ with the combination of ALX and FSK successfully attenuated excessive secretion of proinflammatory cytokines; IL-1 $\beta$, IL-6, and TNF $\alpha$ while, anti-inflammatory IL-10 was secreted in abundance.

By using immunofluorescence to ascertain the locations of GRK5 in $\mathrm{PM}_{\mathrm{F}}$ across all the groups, we explored the possible mode of actions that resulted in the combination therapy being the most potent treatment. We demonstrated in (Fig. 4) that the ALX single therapy inhibited GRK5 expression and prevented it from translocating into the nuclei. Implying that the maladaptive inflammatory responses which still resulted might be due to cancellation of the cAMP-dependent modulation of adaptive inflammatory responses during CCS, just as suggested (Raker VK et al., 2016; Wehbi VL et al., 2016; Bopp T et al., 2009). As shown here (Fig. 4), although GRK5 still translocated into the nuclei of PM $\mathrm{F}_{\mathrm{F}}$-LPS-CCS after treatment with FSK as much as it did without any therapies, inflammatory responses were not aggravated as it did with both ALX single therapy and no therapy groups. Therefore, it is suggested that the combination therapy of ALX and FSK attained its potency mostly via FSK - ACs - cAMP-mediated immunoregulation, coupled with ALX inhibiting GRK5-mediated immune response activation. 
These intriguing outcomes led us to hypothesize that rather than the single therapies of either FSK or ALX, the combination therapy of ALX and FSK, if translated in vivo, may attenuate maladaptive inflammatory response occurring during chronic CCS which drives the adverse remodeling of hearts into pathological cardiac hypertrophy.

We translated the treatments of ALX and FSK in vivo, and at the end of all models, echocardiogram results revealed that the combination of ALX and FSK had effectively preserved cardiac function during CCS with ejection fractions above $65 \%$ and fraction shortenings above $35 \%$ (Fig. 5b, 5c and Supplementary Fig. S4). Again, ALX alone failed to maintain proper function, and although FSK tried to some extent, the echocardiogram of mice treated with only FSK showed all forms of arrhythmias. Protein analysis from apical myocardium was done to elucidate the probable mechanism used by combination therapy to preserve cardiac function during CCS. In summary, we found that even though ALX single therapy successfully inhibited GRK5, cardiac hypertrophy and inflammatory TFs, GATA4, NFAT, MEF2, and NF- $x$ B were still overexpressed during CCS (Fig. 7). This phenomenon also goes to prove Hullmann et al.'s suggestion in 2014, that the inhibition of GRK5 would not halt the activation of NFAT It is also logical then to speculate that the inhibition of GRK5 might not prevent the activation of GATA4, MEF2, and NF- $\chi$ B in vivo, based on our results and the fact that their upregulations and activations interactions with one another. Even so, FSK single therapy decreased the expression of these TFs by upregulating the expression of cAMP (Fig. $6 \mathrm{~b})$.

$\mathrm{PCH}$ mice had dilated left and right heart chambers, excessive cardiomyocyte hypertrophy, and marked deposits of collagen. On the contrary, cardiomyocyte hypertrophy was attenuated by the single treatment with ALX during CCS, although GATA4, NFAT, MEF2, and NF- $x$ B were upregulated. Many others have reported ALX's ability to attenuated myocyte hypertrophy during pressure-over in vitro (Lieu M et al., 2019; Homan KT et al., 2014). However, we are the first to demonstrated that even though single treatment with ALX attenuated cardiac hypertrophy in vivo, neither does it prevent massive collagen deposits nor preserve proper cardiac function during CCS. Also, we demonstrated that FSK single therapy was unable to prevent cardiomyocyte hypertrophy and collagen deposits in the myocardium during CCS, although it did not perform as bad as ALX in the latter. We also set the pace to illustrate that the combination of ALX and FSK, has the therapeutic potential to prevent maladaptive cardiomyocyte hypertrophy and massive interstitial collagen deposits during CCS.

Furthermore, by utilizing CD68 as a biomarker to ascertain the rate of infiltration of macrophages and other mononuclear cells into the myocardium during CCS, we showed that PCH mice had enormous immune cells infiltrating their myocardia in response to myocyte necrosis. Compared to the single therapies of ALX and FSK, their combined therapy was most effective in minimizing the mononuclear cell infiltrations (Fig. 9a). This indicates that the combined therapy prevented of myocyte necrosis and subdued adverse inflammatory responses.

Also, inflammatory cytokine assessment also confirmed that although single treatments with ALX and FSK failed, their combination kept the gap between proinflammatory and anti-inflammatory responses close to a homeostatic immune state. This prevented biased prolonged proinflammatory responses, which could have exacerbated myocyte necrosis and aggravated interstitial collagen deposits. The efficacy of the combination of ALX and FSK in preventing hyperactive inflammatory response and adverse cardiac remodeling during CCS in vivo may be due to the combined efforts of the anti-inflammatory effects they exert individually (Quan MY et al., 2019; Raker VK et al., 2016).

\section{Conclusions}

In summary, we demonstrated in vitro that the combination of ALX and FSK could attenuate LPS-induced hyperactive proinflammatory from $\mathrm{PM}_{\mathrm{F}}$ during CCS. We then induced $\mathrm{PCH}$ in mice and translated the therapies of ALX and FSK in vivo to attenuated the biased proinflammatory immune responses that exacerbate myocyte necrosis and massive collagen deposit during CCS. We investigated the underlying mechanisms of the combination therapy and demonstrated that besides ALX preventing the occurrence of abnormal 
myocytes hypertrophy, it complemented the efforts of FSK - ACs - cAMP-mediated immunoregulation by abolishing GRK5-mediated induction of inflammatory responses (Fig.10). However, singly, neither FSK nor ALX has the therapeutic potential of attenuating PCH by subduing maladaptive inflammatory response while maintaining proper cardiac function during CCS. Also, although ACs and GRKs exhibit a negative correlation in healthy and failing cardiac and immune systems, their mode of actions and pathways are independent of each other.

\section{Limitations}

ALX has the ability to inhibit IkB kinases IKKe and TANK-binding kinase 1 (TBK1) besides GRK5. As such it may be argued that ALX is not a specific inhibitor of GRK. Nonetheless, this study assesses and emphasizes on the phenotypic outcomes of ALX and, or FSK therapies employed in the attempt to prevent the occurrence of PCH during CCS by modulating inflammatory responses.

\section{Acknowledgements}

We appreciate the inputs of Lu Fu during the assessment of cardiac function. This study was funded by the following grants; National Natural Science Foundation of China (No. 81461138036 and No.81370329), Postgraduate Research \& Practice Innovation Program of Jiangsu Province,China (KYCX17-1712);and Priority Academic Program Development of Jiangsu Higher Education Institutions (PAPD)

\section{Author Contributions}

Experiment idea was conceived by the ingenuity of G.K.A. H.S. and G.K.A designed the experiments. G.K. A performed most of the experiments and wrote the manuscript. G.A.K and H.H. isolated and cultured $\mathrm{PM}_{\mathrm{F}}$. A.O.A provided experimental animals. R.R. assisted in making animal models, G.K.A. and T.M. performed sera cytokine analysis. S.Y.A and S.K. performed PMF identification. G.K.A., QM.D. L.K. and X.M. performed cardiac function assessments and G.K.A., S.Y.A, and QM.D. analyzed data. G.K.A., A.O.A., R.R., S.Y.A., M.L.N.N, R.M. and J.O.M. proofread the manuscript.

\section{Competing interests}

The authors declare no competing interests.

\section{References}

1. Paur H, Wright PT, Sikkel MB, Tranter MH, Mansfield C, O'Gara P, et al. (2012). High levels of circulating epinephrine trigger apical cardiodepression in a b2-adrenergic receptor/Gi-dependent manner: a new model of Takotsubo cardiomyopathy. Circulation 126:697-706.

2. Adzika GK, Machuki JO, Shang W, Hou H, Ma T, Wu L, et al. (2019). Pathological cardiac hypertrophy: the synergy of adenylyl cyclases inhibition in cardiac and immune cells during chronic catecholamine stress. J. Mol. Med. 97:897-907.

3. Xie M, Burchfield JS, Hill JA (2013). Pathological ventricular remodeling: therapies: part 2 of 2. Circulation 128:1021-1030.

4. Hartupee J, Mann DL (2016). Role of inflammatory cells in fibroblast activation. J Mol Cell. Cardiol. 93:143-148.

5. Kong P, Christia P, Frangogiannis NG (2014). The pathogenesis of cardiac fibrosis. Cell. Mol. Life. Sci. 71:549-574.

6. Epelman S, Lavine KJ, Beaudin AE, Sojka DK, Carrero JA, Calderon B, et al. (2014). Embryonic and adult-derived resident cardiac macrophages are maintained through distinct mechanisms at steady state and during inflammation. Immunity 40:91-104.

7. Heidt T, Courties G, Dutta P, Sager HB, Sebas M, Iwamoto Y, et al (2014). Differential contribution of monocytes to heart macrophages in steady-state and after myocardial infarction. Circ. Res. 115:284295.

8. Hulsmans M, Sam F, Nahrendorf M (2016). Monocyte and macrophage contributions to cardiac remodeling. J. Mol. Cell. Cardiol. 93:149-155. 
9. Laukova M, Vargovic P, Rokytova I, Manz G, Kvetnansky R (2018). Repeated Stress Exaggerates Lipopolysaccharide-Induced Inflammatory Response in the Rat Spleen. Cell Mol Neurobiol 38:195208.

10. Li Y, Chen L, Kass RS, Dessauer CW (2012). The A-kinase anchoring protein Yotiao facilitates complex formation between adenylyl cyclase type 9 and the IKs potassium channel in heart. J. Biol. Chem. 287:29815-29824.

11. Kipanyula MJ, Woodhoo A, Rahman M, Payne D, Jessen KR, Mirsky R (2013). Calcineurin-nuclear factor of activated $\mathrm{T}$ cells regulation of Krox-20 expression in Schwann cells requires elevation of intracellular cyclic AMP. J. Neurosci. Res. 91:105-115.

12. Pereira L, Rehmann H, Lao DH, Erickson JR, Bossuyt J, Chen J, et al. (2015). Novel Epac fluorescent ligand reveals distinct Epac1 vs. Epac2 distribution and function in cardiomyocytes. Proc. Natl. Acad. Sci. USA 112:3991-3996.

13. Murphy JG, Crosby KC, Dittmer PJ, Sather WA, Dell'Acqua ML (2019) AKAP79/150 recruits the transcription factor NFAT to regulate signaling to the nucleus by neuronal L-type $\mathrm{Ca}^{2+}$ channels. Mol Biol Cell 30:1743-1756.

14. Gerlo S, Kooijman, R, Beck IM, Kolmus, K, Spooren A, Haegeman, G (2011). Cyclic AMP: a selective modulator of NF-xB action. Cell. Mol. Life Sci. 68, 3823-3841.

15. Patial S, Shahi S, Saini Y, Lee T, Packiriswamy N, Appledorn DM, et al. (2011). G-protein coupled receptor kinase 5 mediates lipopolysaccharide-induced NF $x \mathrm{~B}$ activation in primary macrophages and modulates inflammation in vivo in mice. J. Cell. Physiol. 226:1323-1333.

16. Gravning J, Ahmed MS, Qvigstad E, Krobert K, Edvardsen T, Moe IT, et al. (2013). Connective tissue growth factor/CCN2 attenuates-adrenergic receptor responsiveness and cardiotoxicity by induction of G protein-coupled receptor kinase-5 in cardiomyocytes. Mol. Pharmacol. 84:372-383.

17. Hullmann JE, Grisanti LA, Makarewich CA, Gao E, Gold JI, Chuprun JK, et al. (2014). GRK5mediated exacerbation of pathological cardiac hypertrophy involves facilitation of nuclear NFAT activity. Circ. Res. 115:976-985.

18. Quan MY, Song XJ, Liu HJ, Deng XH, Hou HQ, Chen LP, et al. (2019). Amlexanox attenuates experimental autoimmune encephalomyelitis by inhibiting dendritic cell maturation and reprogramming effector and regulatory T cell responses. J Neuroinflammation 16:52.

19. Jia D, Jiang H, Weng X, Wu J, Bai P, Yang W, et al. (2019). Interleukin35 promotes macrophage survival and improves wound healing after myocardial infarction in mice. Circ. Res. 124: 1323-1336.

20. Ray A, Dittel BN (2010). Isolation of Mouse Peritoneal Cavity Cells. J. Vis. Exp. 28:1488.

21. Gold JI, Gao E, Shang X, Premont RT, Koch WJ (2013). Determining the absolute requirement of g protein-coupled receptor kinase 5 for pathological cardiac hypertrophy: Short communication. Circ. Res. 111:1048-1053.

22. Lieu M, Koch WJ (2019). GRK2 and GRK5 as therapeutic targets and their role in maladaptive and pathological cardiac hypertrophy. Expert. Opin. Ther. Targets 23:201-214.

23. Kim MH, Gorouhi F, Ramirez S, Granick JL, Byrne BA, Soulika AM, et al. (2014). Catecholamine stress alters neutrophil trafficking and impairs wound healing by $\beta 2$ adrenergic receptor mediated upregulation of IL-6. J. Invest. Dermatol. 134:809-817.

24. Wu YS, Chen CC, Chien CL, Lai HL, Jiang ST, Chen YC, et al. (2017). The type VI adenylyl cyclase protects cardiomyocytes from $\beta$-adrenergic stress by a PKA/STAT3-dependent pathway. J. Biomed. Sci.24:68.

25. Tang T, Gao MH, Lai NC, Firth AL, Takahashi T, Guo T, et al. (2008). Adenylyl cyclase type 6 deletion decreases left ventricular function via impaired calcium handling. Circulation 117:61-69.

26. Raker VK, Becker C, Steinbrink K (2016) The cAMP pathway as therapeutic target in autoimmune and inflammatory diseases. Front. Immunol. $7: 123$.

27. Premont RT, Gainetdinov RR (2007). Physiological roles of G protein-coupled receptor kinases and arrestins. Annu. Rev. Physiol. 69:511-534.

28. Martini JS, Raake P, Vinge LE, DeGeorge BR Jr, Chuprun JK, Harris DM, et al. (2008) Uncovering $\mathrm{G}$ protein-coupled receptor kinase- 5 as a histone deacetylase kinase in the nucleus of cardiomyocytes. 
Proc. Natl. Acad. Sci. USA 105:12457-12462.

29. Islam KN, Bae JW, Gao E, Koch WJ (2013). Regulation of nuclear factor $x \mathrm{~B}(\mathrm{NF}-x \mathrm{~B})$ in the nucleus of cardiomyocytes by G protein-coupled receptor kinase 5 (GRK5). J. Biol. Chem. 288:35683-35689.

30. Sorriento D, Santulli G, Ciccarelli M, Maione AS, Illario M, Trimarco B, et al. (2018). The Amino-Terminal Domain of GRK5 Inhibits Cardiac Hypertrophy through the Regulation of CalciumCalmodulin Dependent Transcription Factors. Int. J. Mol. Sci. 19:861.

31. Zimmer A, Bagchi AK, Vinayak K, Bello-Klein A, Singal PK (2019). Innate immune response in the pathogenesis of heart failure in survivors of myocardial infarction. Am J. Physiol. Heart. Circ. Physiol. 316:H435-H445.

32. Liu YZ, Wang YX, Jiang CL (2017). Inflammation: The Common Pathway of Stress-Related Diseases. Front. Hum. Neurosci. 11:316.

33. Maydych, V (2019). The Interplay Between Stress, Inflammation, and Emotional Attention: Relevance for Depression. Front. Neurosci.13 :384.

34. Packiriswamy N, Parameswaran N (2015). G-protein-coupled receptor kinases in inflammation and disease. Genes Immun. 16:367-377.

35. Wehbi VL, Taskén K (2016). Molecular Mechanisms for cAMP-Mediated Immunoregulation in T cells - Role of Anchored Protein Kinase A Signaling Units. Front. Immunol. 7:222.

36. Bopp T, Dehzad N, Reuter S, Klein M, Ullrich N, Stassen M, et al. (2009). Inhibition of cAMP degradation improves regulatory T cell-mediated suppression. J. Immunol. 182:4017-4024.

37. Homan KT, Wu E, Cannavo A, Koch WJ, Tesmer JJ (2014). Identification and characterization of amlexanox as a G protein-coupled receptor kinase 5 inhibitor. Molecules 19:16937-16949.

\section{Hosted file}

British J Pharma_Figures_PCH_Therapeutic intervention.docx available at https://authorea. com/users/347381/articles/473105-combination-of-amlexanox-and-forskolin-attenuates-

pathological-cardiac-hypertrophy-by-subduing-maladaptive-inflammatory-response 